\title{
Hair Diameter Variation in Different Vertical Regions of the Occipital Safe Donor Area
}

\author{
Seon Sik Yun ${ }^{1}$, Jae Hyun Park ${ }^{2}$, Young Cheon $\mathrm{Na}^{1}$ \\ ${ }^{1}$ Department of Plastic and Reconstructive Surgery, Wonkwang University School of Medicine, Iksan; ${ }^{2}$ Dana Plastic Surgery Clinic, Seoul, \\ Korea
}

Background Little is known concerning hair diameter variation within the safe donor area for hair transplantation surgery. Thicker or thinner hair may be needed, depending on the recipient area, hairline design, and the purpose of surgery.

Methods Twenty-seven patients ( 7 men and 20 women; mean age, 28 years; range, 20-47 years) were included in this study. The midoccipital point was used as the reference point on the horizontal plane at the upper border of the helical rim. The target area width was $15 \mathrm{~cm}$ ( $7.5 \mathrm{~cm}$ to the right and left of the reference point) and the height was $8 \mathrm{~cm}(2 \mathrm{~cm}$ above and $6 \mathrm{~cm}$ below the reference point). The study area was divided horizontally into $35-\mathrm{cm}$ sections $(A, B, C)$ and vertically into 4 2-cm sections (1-4), creating a total of 12 zones. Ten anagen hairs were randomly obtained from each zone and their diameters were measured.

Results Hair diameter in the 4 vertical sections varied significantly, gradually decreasing from sections 1 (superior) to 4 (inferior) in all 3 horizontal sections ( $A, B$, and $C$ ).

Conclusions Our results suggest that sections 1 and 2 of the occipital safe donor area would be useful for obtaining thicker hair, such as in procedures to treat male- and female-pattern hair loss, whereas hair from zones 3 and 4 could be useful for transplantation surgery requiring thinner hair, such as eyebrows, eyelashes, and female hairline correction. Our results may be clinically valuable for planning hair transplant surgery and choosing the optimal donor region.

\section{Keywords Hair follicle / Transplantation / Transplant donor site}

Correspondence: Jae Hyun Park Dana Plastic Surgery Clinic, 10F Samju Building, 606 Gangnam-daero, Gangnam-gu, Seoul 06038, Korea Tel: +82-2-512-0922

Fax: +82-2-512-0942 E-mail: jay8384@naver.com
No potential conflict of interest relevant to this article was reported.

\section{INTRODUCTION}

The definition of the safe donor area (SDA) is one of the most important theories in the history of modern hair transplantation. First introduced by Unger et al. [1] in 1994, most hair surgeons consider the SDA to be the standard theory of transplantation.

Hair diameter is the most crucial prognostic factor in deter- mining the results of surgery [2]. Transplanting thicker-diameter hair obviously results in fuller volume. However, narrow-caliber hair is crucial for achieving a soft and natural appearance in eyebrow, eyelash, and female hairline correction operations. In particular, follicular unit extraction (FUE) allows the surgeon to selectively punch and extract thicker or thinner hairs according to the operative plan.

Generally, hair in the nape and periauricular region is thinner 
than in other areas [3]. However, little is known about the vertical location at which hair caliber starts to narrow, or in which areas hair retains its thickness.

Differences in hair diameter according to the location within the donor area are of great clinical importance and significantly influence operative planning pertaining to donor site issues such as surgical method and design. Therefore, we report the results of our study of hair diameter in the SDA and present a review of the literature.

\section{METHODS}

A total of 27 volunteers ( 7 men and 20 women) participated in this study. Written informed consent was obtained from all participants. Participants were Korean and had black hair. Patient age ranged from 20 to 47 years, with a mean of 28 years. Those with a history of previous hair transplantation surgery, hair disease, use of finasteride, or face-lift surgery were excluded.

The midoccipital point on the horizontal plane at the upper border of the helical rim was set as the reference point. The study area width was $15 \mathrm{~cm}$, extending $7.5 \mathrm{~cm}$ bilaterally from the midpoint; the height was $8 \mathrm{~cm}, 2 \mathrm{~cm}$ above and $6 \mathrm{~cm}$ below the reference point. This area was then divided into 12 zones, each $5 \mathrm{~cm}$ wide $\times 2 \mathrm{~cm}$ high (Fig. 1).

Ten anagen hairs were randomly selected from each of the 12 zones and their diameter was evaluated. A Folliscope (LeadM, Seoul, Korea) was used for all measurements. Anagen hairs were screened by a highly experienced hair surgeon for accuracy. Hair thickness was assessed by measuring shaft diameter within $1 \mathrm{~cm}$ of the scalp skin.

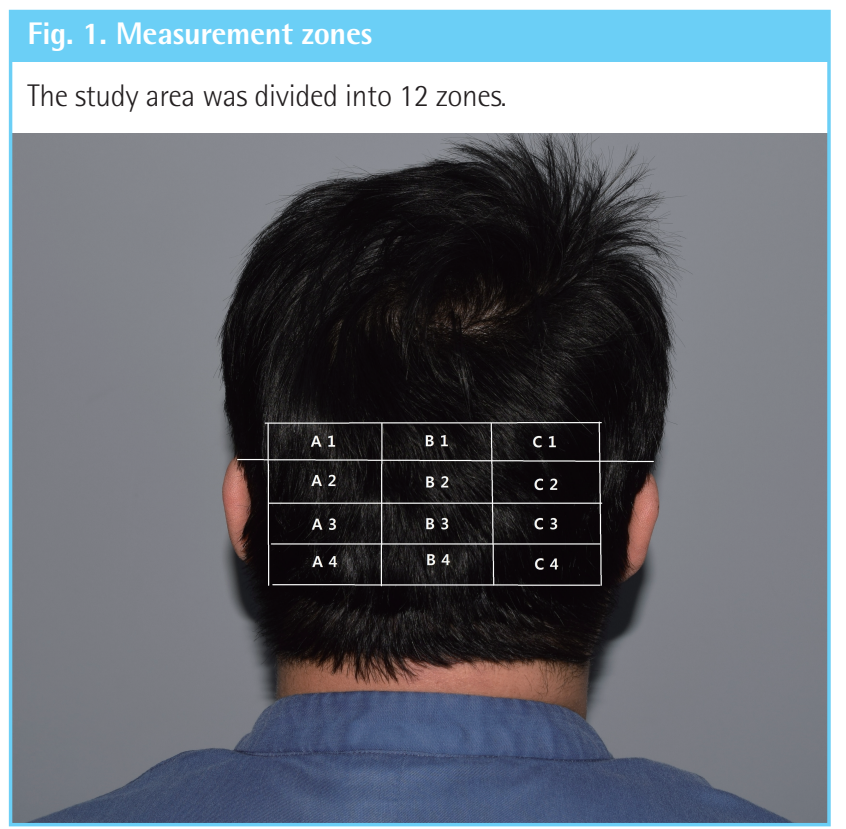

\section{Statistics}

SPSS for Windows ver. 21.0 (IBM Corp., Armonk, NY, USA) was used for statistical analysis. Differences in hair thickness by zone were evaluated with 1-way analysis of variance (ANOVA). Differences among groups were calculated using the Scheffé test.

\section{RESULTS}

A significant difference in hair diameter was found among vertical sections $1,2,3$, and 4 , with the diameter gradually decreasing from section 1 to section 4 in all 3 horizontal sections (A, B, and C). The average hair thickness values are shown in Table 1 .

\section{Differences in hair caliber among vertical sections 1 to 4} One-way ANOVA was used to evaluate differences in hair thickness from section 1 to section 4 in each horizontal area (A, B, and $C$ ). The Scheffé test was used to determine differences among vertical sections according to the horizontal section.

Significant differences were found between A1 and A2, A3, and $\mathrm{A} 4(\mathrm{~A} 1>\mathrm{A} 2, \mathrm{~A} 3, \mathrm{~A} 4)(\mathrm{P}<0.001)$ (Table 2), and between $\mathrm{B} 1$ and $\mathrm{B} 2, \mathrm{~B} 3$, and B4 $(\mathrm{B} 1>\mathrm{B} 2, \mathrm{~B} 3, \mathrm{~B} 4)(\mathrm{P}<0.001)$ (Table 3$)$.

\section{Table 1. Average hair thickness}

\begin{tabular}{|lccc|}
\hline Zone & A & B & C \\
\hline 1 & 0.0816 & 0.0816 & 0.0816 \\
2 & 0.0764 & 0.0761 & 0.0764 \\
3 & 0.0733 & 0.0736 & 0.0733 \\
4 & 0.0691 & 0.0692 & 0.0691 \\
\hline Unit, mm. & & & \\
\hline
\end{tabular}

\begin{tabular}{|lccccc|}
\hline \multicolumn{7}{|l}{ Table 2. Hair thickness differences in section A } \\
\hline Zone & No. & Mean & $\begin{array}{c}\text { Standard } \\
\text { deviation }\end{array}$ & F/P-value & Scheffe \\
\hline A1 & 27 & 0.0816 & 0.0062 & $24.221 /$ & A1 > A2,A3,A4 \\
A2 & 27 & 0.0764 & 0.0049 & $(<0.001)$ & \\
A3 & 27 & 0.0733 & 0.0056 & & \\
A4 & 27 & 0.0691 & 0.0055 & & \\
\hline Unit, $\mathrm{mm}$. & & & & & \\
\hline
\end{tabular}

\section{Table 3. Hair thickness differences in section B}

\begin{tabular}{|lccccc|}
\hline Zone & No. & Mean & $\begin{array}{c}\text { Standard } \\
\text { deviation }\end{array}$ & F/P-value & Scheffe \\
\hline B1 & 27 & 0.0816 & 0.0068 & $21.539 /$ & B1>B2,B3,B4 \\
B2 & 27 & 0.0761 & 0.0052 & $(<0.001)$ & \\
B3 & 27 & 0.0736 & 0.0057 & & \\
B4 & 27 & 0.0692 & 0.0053 & & \\
\hline Unit, mm. & & & & & \\
\hline
\end{tabular}




\begin{tabular}{|l} 
Table 4. Hair thickness differences in section C \\
\begin{tabular}{|lccccc|}
\hline Zone & No. & Mean & $\begin{array}{c}\text { Standard } \\
\text { deviation }\end{array}$ & F/P-value & Scheffe \\
\hline C1 & 27 & 0.0800 & 0.0080 & $12.796 /$ & C1 > C3,C4 \\
C2 & 27 & 0.0774 & 0.0048 & $(<0.001)$ & C2 $>$ C4 \\
C3 & 27 & 0.0738 & 0.0052 & & \\
C4 & 27 & 0.0707 & 0.0051 & & \\
\hline Unit, mm. & & & & & \\
\hline
\end{tabular}
\end{tabular}

Significant differences were found between $\mathrm{C} 1$ and $\mathrm{C} 3$ and $\mathrm{C} 4$ $(\mathrm{C} 1>\mathrm{C} 3, \mathrm{C} 4)$, and between $\mathrm{C} 2$ and $\mathrm{C} 3$ and $\mathrm{C} 4(\mathrm{C} 2>\mathrm{C} 3, \mathrm{C} 4)$ $(\mathrm{P}<0.001)$ (Table 4).

The vertical sections $1,2,3$, and 4 showed significant differences, with the hair diameter decreasing from section 1 to section 4 in all 3 horizontal sections $(\mathrm{A}, \mathrm{B}$, and $\mathrm{C})(\mathrm{P}<0.001)$.

\section{Differences in hair thickness among horizontal sections $A, B$, and C}

One-way ANOVA and the Scheffé test did not reveal any statistically significant differences in hair thickness among sections A, $\mathrm{B}$, and $\mathrm{C}(\mathrm{F}=0.467, \mathrm{P}>0.05)$.

\section{DISCUSSION}

Hair transplantation surgery fundamentally requires donor harvesting from the SDA. The SDA was first suggested by Unger et al. [4] in 1994 and continues to be the global standard .

The SDA is defined by 3 boundaries [5]. The anterior boundary coincides with a vertical line drawn at the external acoustic meatus. The inferior boundary is controversial, and may move upward with time. The superior border depends on the extent of vertex alopecia, and therefore becomes the most crucial component in male-pattern baldness. Park, in particular, has previously claimed that the position of the parietal whorl and vertex alopecia have a close relationship with the SDA [6].

Cole described the SDA that is applicable to FUE procedures [7]. He determined the permanent donor area to be $203 \mathrm{~cm}^{2}$, and divided this region into 8 major zones $(3.5 \times 6 \mathrm{~cm})$ and 6 minor zones $(3.5 \times 2 \mathrm{~cm})$. Considering the possibility that the further extension of vertex alopecia can cause the superior border to move downwards, the 2 major zones in the center were reduced in height to $3.5 \times 5 \mathrm{~cm}$.

In the present study, the occipital donor area was divided vertically into 2-cm blocks to include both major and minor zones. A Folliscope (LeadM, Seoul, Korea) was used to measure hair diameter. The Folliscope is relatively simple to use and allows the accurate evaluation of hair thickness [8].

Our results indicate that within the occipital donor area, hair tended to be narrower in lower sections, with an average difference of 10 microns between sections 1 and 4 .

These results are very helpful in operative planning and in strip surgery design. Generally, in patients with alopecia, strip surgery is primarily performed in the area of highest density within the SDA. However, if thinner-caliber hair is required, it could be more beneficial to harvest the donor grafts as low on the scalp as possible. Moreover, the occipital protuberance is located in zone B3 according to the classification used in this study. That is, the occipital protuberance is found 2 to $4 \mathrm{~cm}$ below the upper border of the helical rim on the horizontal plane. Scalp laxity is lower below the occipital protuberance. Moreover, the insertion of the nape muscles may cause more postoperative pain. Therefore, it is crucial to foresee all the following factors during operative planning: the number of required grafts, 1-hair follicular units, and thin hairs, with identification of the laxity, elasticity, glidability, design, and location of the area to be harvested, and the direction and thickness of the original hair at the preexisting frontal hairline [9]. Accordingly, the surgeon should carefully determine the harvesting area, width, length, design, and surgical methods.

This information is also useful in conducting FUE procedures. FUE has the advantage that the surgeon can selectively harvest donor hairs from a relatively large donor area. Fully shaving the donor area can be beneficial, but partial shaving will suffice when full shaving is not an option. Partial shaving is done in the form of a patch box; in this case, the location of shaving determines the thickness of the extracted hairs. The entire donor area can be used for harvesting in non-shaven FUE, allowing focused harvesting according to the hair thickness that is needed [10].

The recipient area can be anywhere on the body, including the eyebrow, eyelash, pubic area, hairline, vertex, sideburns, or beard. Moreover, there are many different purposes for hair transplantation. Although the most common reason for hair transplantation is to treat alopecia, hair transplantation is often done to achieve a more feminine, slimmer, and more attractive appearance in female hairline correction surgery [11-13], or to obtain a sexy, masculine appearance in beard creation. Hair transplantation can also be used to cover scalp scars resulting from various plastic surgery procedures. Different recipient areas and operative purposes require donor hairs of diverse calibers.

The surgical skills involved in hair transplantation have become increasingly precise and delicate in parallel with patient demands. Occipital donor hairs are generally thicker than frontal hairs. Therefore, extra effort is required to obtain thin hair when doing so is necessary. For example, female hairline correction surgery does not simply involve transplanting 1-hair follicu- 
lar units; 1-hair follicular units must be further divided into vellus hairs, very thin single hairs, and thick single hairs according to the recipient site to achieve a more natural look with appropriate gradation. In particular, side hairline correction surgery requires 1-hair follicles, and greater numbers of thinner hairs are needed [11].

When adequate thin hairs are not obtained in hairline correction surgery, very awkward surgical results can occur, sometimes requiring laser treatment to thin the transplanted hairs $[13,14]$. Eyebrow and eyelash transplantation are good examples of situations where thin hairs are required as much as possible. However, such cases only require 100 to 300 hairs at once. Therefore, when proceeding with strip surgery, the donor site can be designed at a much inferior location or on the temporal side.

In contrast, when performing female hairline correction surgery, in which relatively large numbers of donor hairs are required, including many thin hairs, the location of the donor strip can vary by case according to the recipient site and the number of hair follicles needed. When harvesting by FUE, hairs extracted from a superior portion of the donor area can be better used at the back of the hairline, while those from an inferior portion can be used at the very front or side hairline area.

In general, nape hairs and those from the minor zones are thinner in caliber than those in the major SDA zones. However, to the authors' knowledge, little has been reported about differences in hair thickness within the major SDA zones. We are confident that this report will provide clinically valuable information to hair surgeons.

As many thick hairs as possible are needed in procedures to treat male pattern baldness, female pattern hair loss, and other hair transplantation operations performed to remedy alopecia. There is currently more to hair transplantation than planting as many hair follicles as possible with dense packing. Delicate techniques utilizing thicker and thinner hairs from the same number of grafts are needed to obtain a more voluminous, soft, and natural appearance.

Our study showed that hair thickness in the occipital donor area gradually decreased from superior to inferior in both sexes. The hair diameter was especially narrow ( $<70$ microns) in section 4. Park has commented that the target thickness of thin hair laser therapy is 65 microns for hairline correction surgery [15]. In the present study, there was a difference of approximately 10 microns in hair thickness between section 1 and section 4 . Such a difference can be crucial in female hairline correction surgery.

Therefore, when performing strip surgery for female hairline correction surgery in Asian women, we suggest performing, in parallel, some FUE harvesting in section 4 for use in the very foremost part of the hairline. Moreover, when exclusively per- forming FUE procedures, it may be helpful to extract thinner hairs from below the occipital protuberance for use at the very front or side hairline area. One should keep in mind that harvesting in this area has a higher transection rate because of the more acute hair exit angle. In contrast, for cases requiring as many thick hairs as possible, harvesting from sections 1 and 2 is recommended.

\section{REFERENCES}

1. Unger W, Solish N, Giguere D, et al. Delineating the "Safe" donor area for hair transplanting. Am J Cosmet Surg 1994; 11:239-43.

2. Devroye J. An overview of the donor area: basic principles. In: Unger WP, Zari S, editors. Hair transplantation. 5th ed. New York: Informa Healthcare; 2011. p.247-62.

3. Umar S. Use of nape and peri-auricular hair by follicular unit extraction to create soft hairlines and temples: my experience with 128 patients. Aesthet Surg J 2015;35:903-9.

4. Unger W, Solish N, Giguere D, et al. Delineating the "Safe" donor area for hair transplanting. Am J Cosmet Surg 1994; 11:239-43.

5. Rassman WR, Carson S. Micrografting in extensive quantities: the ideal hair restoration procedure. Dermatol Surg 1995;21:306-11.

6. Park JH, Na YC, Moh JS, et al. Predicting the Permanent safe donor area for hair transplantation in Koreans with male pattern baldness according to the position of the parietal whorl. Arch Plast Surg 2014;41:277-84.

7. Jimenez F, Ruifernandez JM. Distribution of human hair in follicular units: a mathematical model for estimating the donor size in follicular unit transplantation. Dermatol Surg 1999;25:294-8.

8. Lee BS, Chan JY, Monselise A, et al. Assessment of hair density and caliber in Caucasian and Asian female subjects with female pattern hair loss by using the Folliscope. J Am Acad Dermatol 2012;66:166-7.

9. Park JH. Association between scalp laxity, elasticity, and glidability and donor strip scar width in hair transplantation and a new elasticity measuring method. Dermatol Surg 2017;43:574-81.

10. Park JH. Direct non-shaven FUE technique. Hair Transplant Forum Int 2014;24:103-4.

11. Park JH. Side-hairline correction in Korean female patients. Plast Reconstr Surg Glob Open 2015;3:e336.

12. ParkJH. Novel principles and techniques to create a natural design in female hairline correction surgery. Plast Reconstr Surg Glob Open 2015;3:e589. 
13. Park JH. Masking the close eye appearance in the east asian female population: infratemporal hairline reduction with hair grafting. Aesthetic Plast Surg 2016;40:921-5.

14. Park HS, Kim JY, Choe YS, et al. Alternative method for creating fine hairs with hair removal laser in hair transplanta- tion for hairline correction. Ann Dermatol 2015;27:21-5.

15. Park JH, Lee SY, You SH, et al. Photo epilation with intense pulsed light for thinning of anterior hairline after hairline correction surgery in east asians. Arch Plast Surg 2017;44: $157-61$. 